\title{
Las innovaciones pedagógicas y la gestión de la educación en la Escuela Nueva Laboratorio “Emma Gamboa” de la Universidad de Costa Rica y el Instituto Educativo Moderno
}

\author{
Pedagogical innovations and management of education in Escuela Nueva Laboratorio "Emma Gamboa" of \\ University of Costa Rica and Instituto Educativo Moderno
}

Recibido 15 enero 2015 • Aceptado 28 mayo 2015 • Corregido 01 junio 2015

\author{
Milena Brenes Montero' \\ Instituto Educativo Moderno \\ San José, Costa Rica \\ milebre@hotmail.com
}

\begin{abstract}
Resumen. El presente artículo pretende analizar el desarrollo de innovaciones pedagógicas desde la gestión en la Escuela Nueva Laboratorio "Emma Gamboa" de la Universidad de Costa Rica y en el Instituto Educativo Moderno, a partir de la identificación de los mecanismos para la implementación de estas innovaciones, la determinación de las estrategias desarrolladas por docentes en esta área y el establecimiento de las acciones de seguimiento, aplicadas desde la gestión, en el desarrollo de estas innovaciones. La investigación, de tipo descriptivo, se ejecutó entre los meses de octubre y diciembre del año 2013, con la participación de las dos directoras y el personal docente de ambos centros educativos, a quienes se les aplicó un cuestionario estructurado de acuerdo con las categorías de análisis definidas. Entre los principales hallazgos encontrados se señala que desde la fundación de ambas escuelas se procura la implementación de innovaciones pedagógicas, identificándose a su vez, áreas susceptibles de mejora, tanto en la implementación como la ejecución y el seguimiento de estas.
\end{abstract}

Palabas clave. Innovación; pedagogía; gestión de la educación

\begin{abstract}
The present article meets the general objective: analyze the development of pedagogical innovations from the management in Escuela Nueva Laboratorio "Emma Gamboa" of University of Costa Rica and Instituto Educativo Moderno. The proposed specific objectives were: identify the arrangements that the management applies to implement pedagogical innovations; define the strategies developed by teachers in this area; and establish the follow up actions, applied from the management, in the develop of this innovations. This is a quantitative with a descriptive approach, it was executed between October and December of 2013. The subjects of investigation were the
\end{abstract}

1 Máster en Administración Educativa de la Universidad de Costa Rica. Bachiller en Ciencias de la Educación con énfasis en Educación Preescolar de la Universidad de Costa Rica. Creadora e implementadora de metodología para lectoescritura con enfoque constructivista (atención a la diferencia: dificultad y talento). Corresponsable de diseño e implementación de metodología de trabajo del preescolar desde Teoría de Inteligencias Múltiples. Actualmente labora como maestra de educación primaria en el Instituto Educativo Moderno, San José, Costa Rica. 
two principals and the teaching staff of both education centers. The instruments used were two questionnaires, one directed to teachers and the other one directed to the principals of both schools, structured according with the analysis categories defined. Among the principal discoveries found, it's important to note that since the foundation of this schools they attempts the implementation of pedagogical innovations, identifying at the same time, susceptible areas of improvement in the implementation as well as in the execution and the follow up of them.

Keywords. Innovation; pedagogy; management of education

\section{Introducción}

La educación es la responsable del proceso socializador que determina a un grupo humano particular. Es, además, la principal herramienta para alcanzar desarrollo personal y grupal. Tiene, por lo tanto, el poder de transformar. Con la claridad de su importancia, estados y organismos no gubernamentales, han fijado metas y proyectado objetivos para garantizar el rumbo que este proceso debe seguir, tanto en el nivel macro como en el regional.

Surgen, en este contexto, diversas iniciativas que buscan la integración de países y regiones para el logro de metas que, a escala mundial, mejoren las condiciones de vida de los pueblos. Entre estas se pueden citar: la Conferencia Mundial sobre Educación para Todos celebrada en Jomtien (Organización de las Naciones Unidas para la Educación, la Ciencia y la Cultura [UNESCO], 1990), el Foro Mundial sobre Educación celebrado en el Marco de Acción de Dakar (UNESCO, 2000), la Cumbre del Milenio realizada en la sede de las Naciones Unidas (2000) donde se establecen los Objetivos de Desarrollo del Milenio, así como la iniciativa La educación ante todo gestada por la Oficina del Secretario General de la Organización de las Naciones Unidas (ONU, 2012). Sin embargo, no pueden entenderse estas propuestas de forma plena sin tomar en cuenta la realidad socioeconómica que caracteriza al mundo globalizado actual.

En América Latina, la experiencia en cuanto al desarrollo de la educación no es ajena al proceso que se genera en el resto de las áreas, permeadas todas por los cambios impulsados en el nivel económico. Como señala Chomsky, citado por López y Flores (2006):

No es posible conocer ni explicar los objetivos de los programas y las políticas públicas de Latinoamérica, sin considerar las recomendaciones de las agencias internacionales de financiamiento, que detallan específicamente cada área de la vida de los países no desarrollados, condicionando los empréstitos al cumplimiento de sus recomendaciones. (p. 2)

De manera que las prioridades están determinadas por los lineamientos neoliberales internacionales, de acatamiento obligatorio, que llevan a los países a definir el alcance de áreas como las de servicio, incluida la educación.

Esta priorización, aplicada en América Latina, ha impactado básicamente: 
- En la economía política del financiamiento educativo.

- En las vinculaciones entre educación y trabajo.

- En la creación de estándares de excelencia académica internacional, con sus implicaciones a nivel de evaluación del currículum, educación superior o formación docente. (Torres, 2008, pp. 219-220)

Desde esta racionalidad se conceptualiza el término de calidad en el proceso educativo, referida, de forma puntual, a parámetros de excelencia académica según lineamientos internacionales, dejando de lado el resto de elementos que conlleva la educación.

En concordancia, Aubert y García (2009) plantean que "el neoliberalismo en el ámbito educativo ha sido visto como un instrumento a partir del que las clases dominantes o la cultura hegemónica se han impuesto priorizando las demandas del mercado a las necesidades humanas" (p. 233), por lo que la definición del proceso educativo en áreas como la innovación, se ve permeado por la fuerza e intereses de esas demandas.

Resulta imperante, por lo tanto, definir el concepto de innovación que se promueve y que rige las acciones en el ámbito educativo.

Las innovaciones pueden ser concebidas como oportunidades de convertir al proceso educativo en pertinente y oportuno, según las demandas externas. Esto sin perder la dimensión del compromiso con el desarrollo personal del ser humano que lo vive, trascendiendo aspectos aislados de tipo temático o programático, para aspirar a transformar la pedagogía misma que lo sostiene. De forma tal que se plantea la disyuntiva entre responder a la racionalidad dominante o generar un análisis de esta y, por ende, formas alternativas de desarrollo del proceso educativo.

En este sentido, se entiende innovar como:

La búsqueda autónoma de nuevos caminos, induciendo al cambio de mentalidad, de las representaciones y de los estereotipos, posibilitando el desarrollo de la capacidad crítica, de la autorreflexión e interpretación de las prácticas pedagógicas hasta llegar a una nueva comprensión del mundo como manera de creación de una nueva conciencia. (Bunes, Cánovas, Ruiz y López, 2009, p. 141)

Por lo que innovar requiere de un proceso de análisis amplio, que trascienda acciones aisladas dentro del aula, la ampliación de contenidos en programas o, incluso el uso de TIC's. Puede, por lo tanto, innovarse desde diferentes concepciones. Como apunta Tack, citado por Moreno (1997), se pueden diferenciar dos tipos de innovaciones, las instrumentales y las referidas a los fines:

Las innovaciones referidas a los fines pedagógicos, son aquellas que crean nuevas posibilidades pedagógicas y hacen alcanzables nuevas finalidades de formación y educación escolares (...), las innovaciones instrumentales, solo procuran medios de realización más efectivos y más económicos para alcanzar ideas finalistas. (p. 47) 
De manera tal que innovar en el plano de las finalidades, es apuntar al cambio y transformación en el campo de la pedagogía, analizando e innovando desde los objetivos de formación, abarcando la integralidad del proceso. Es desde este planteamiento que las innovaciones pedagógicas pueden generar cambios en la función misma de la educación.

En Costa Rica, en el marco de los documentos oficiales emanados desde la Asamblea Legislativa de la República, el Consejo Superior de Educación y el Ministerio de Educación Pública, se establece la importancia del carácter innovador que comprende el proceso educativo. El Consejo Superior de Educación (1994), en la Política educativa hacia el siglo XXI, plantea la importancia de las innovaciones en la educación, señalando que:

La investigación educativa, en el ámbito nacional, provincial, regional e institucional, debe constituirse en una de las fuentes primordiales para sustentar la continuidad e innovación de las acciones educativas tanto en la práctica cotidiana del aula como en la toma de decisiones administrativas. (p. 3)

Se cuenta con fundamento para propiciar las innovaciones, dando por sentado que estas deben darse, constituyéndose en una característica de la práctica cotidiana del aula, entiéndase modelo pedagógico, y de las prioridades que guían las decisiones desde lo administrativo. A este respecto, Pinto (2010) señala que:

En el caso de la educación Latinoamericana todavía la innovación pedagógica y curricular no es una cultura vigorosa; por el contrario, comprobamos lo difícil que resulta innovar precisamente por los obstáculos de todo tipo que colocan las clases políticas a todo lo que no sea homogeneizar resultados y estandarizar conductas y prácticas docentes. (p. 79)

Se evidencia que las posturas o discursos filosóficos, contenidos en los documentos que han generado las últimas políticas educativas en los países latinoamericanos, incluida Costa Rica, no están igualmente respaldados por acciones y determinaciones concretas que produzcan innovaciones pedagógicas que permeen desde los lineamientos oficiales hasta las prácticas en las aulas.

De esta manera se definen los siguientes objetivos para esta investigación:

\section{Objetivo general}

- Analizar el desarrollo de innovaciones pedagógicas desde la gestión en la Escuela Nueva Laboratorio Emma Gamboa de la Universidad de Costa Rica y en el Instituto Educativo Moderno.

\section{Objetivos específicos}

- Identificar los mecanismos que aplica la gestión para la implementación de innovaciones pedagógicas en las organizaciones educativas. 
- Determinar las estrategias de innovación pedagógica desarrolladas por los docentes en las organizaciones educativas.

- Establecer las acciones de seguimiento, aplicadas desde la gestión, en el desarrollo de innovaciones pedagógicas en las organizaciones educativas.

\section{Referentes conceptuales}

\section{Conceptualización de la educación}

La educación es un proceso complejo que implica diferentes áreas de la vida social e individual del ser humano. Transcurre desde lo informal de la vida en sociedad, hasta lo formal e institucional. Es inherente a la vida misma de las personas, por lo tanto, su definición no es simple, puede señalarse que en cualquiera de sus manifestaciones, "es hacer salir y manifestarse las facultades y potencialidades que cada individuo tiene en su ser personal" (Dengo, 2001, p. 5).

Es una actividad que se desarrolla desde la relación con otros. Como señala Freire, citado por Rodríguez, Marín, Moreno y Rubano (2007), la educación es "primeramente comunicación, diálogo entre iguales que se relacionan; no es meramente una acción comunicativa que tiende al consenso sino que es una acción estratégica que apunta a la consecuencia de un fin, que es la transformación del mundo" (p. 136). Por lo que, el carácter activo que trasciende a la experiencia particular de aprendizaje, genera trasmisión, en una búsqueda de una significación conjunta. Es un proceso que no culmina en el plano de lo individual, su máxima expresión se concreta y completa con la construcción conjunta.

Al margen de las bases filosóficas en que se fundamente el proceso educativo y en el contexto actual de globalización, entran en juego diversas variables para determinar, en la praxis, el rumbo que la educación y todos sus componentes, incluida la calidad, deben seguir.

Delimitada por las condiciones político-económicas que definen al sistema social en su conjunto, la calidad de la educación es conceptualizada y operacionalizada desde planteamientos específicos, derivados de esta lógica. Así, por ejemplo, en el caso de Costa Rica, se plantea que "la sostenibilidad económica y productiva representa, para la educación, el reto de generar los recursos humanos necesarios para elevar la competitividad y productividad nacionales e integrar exitosamente el país a la economía mundial" (Consejo Superior de Educación, 1994, p. 5), definiendo una relación determinante entre criterios de mercado y objetivos de la educación, por lo que el alcance de calidad estará directamente vinculado con estos nuevos fines.

Por lo tanto, los parámetros definidos para evaluar la calidad responden a criterios impuestos por los grandes organismos financieros, como el Fondo Monetario Internacional o el Banco Mundial. Estos organismos, cuya naturaleza y especialidad es la económica, definen 
que "el objetivo final de la política educativa es la eficiencia económica, la liberalización de los mercados y la globalización del capital, todo lo cual lleva a un sobre énfasis en métodos cuantitativos para medir el éxito" (Torres, 2008, p. 219), delimitando la calidad a estándares para la evaluación del aprendizaje y de los sistemas educativos mismos reducidos a logros en el área de los contenidos y al alcance de destrezas que permitan insertarse en el mercado laboral.

Al respecto, Ramírez (2012), plantea que deben considerarse varios aspectos, al tratar de definir la calidad de la educación, señalando como imprescindibles los siguientes: "rendimiento académico del estudiantado, formación del equipo de profesores, nivel socioeconómico de los padres y madres de familia o encargados, condiciones socioculturales de la niñez y elaboración de políticas públicas con compromiso social y criterios de expertos" (p. 7). Por lo tanto, para definir calidad debe analizarse más que el rendimiento registrado en pruebas académicas.

\section{Gestión de la educación}

Gestión y administración en el área de la educación son procesos que apuntan a niveles diferentes de acción y ejecución. Así, Aguerrondo, Lugo, Pogré, Rossi, Xifra (2006) explican que:

Administración nos remite a dinamizar lo existente, sin salir del paradigma, en el marco de las teorías clásicas de la organización; mientras que gestión nos remite a una serie de procesos de transformación que se dan en la organización educativa, con vistas a cambiar el paradigma. (p. 45)

De manera que la gestión incluye al proceso administrativo, implicando trascenderlo en lo referente al cumplimiento de pasos o fases establecidas, para proyectarse a la construcción y creación de nuevos elementos. No obstante, existen distintas construcciones de gestión, derivadas de planteamientos particulares de elementos como el concepto de ser humano, la teoría de sociedad y la filosofía de ciencia, entre otros, que producen la conceptualización y desarrollo de un específico tipo de educación con su respectiva determinación de calidad de vida (Sander, 2002).

Esta amplitud de acción que comprende la gestión, contiene cuatro dimensiones de acción, a saber la institucional, la administrativa, la comunitaria y la pedagógica (UNESCO, 2011).

Dentro de la dimensión institucional se comprenden la estructura de la organización, las instancias y responsabilidades de cada actor que conforma la institución, con sus normas explícitas e implícitas. La dimensión administrativa incluye lo relativo al manejo de recursos, tanto materiales y económicos como humanos, especifica el cumplimiento de la normativa y la supervisión de funciones. La dimensión comunitaria es la relativa a relaciones con las familias, el entorno inmediato y la comunidad. La dimensión pedagógica atiende lo referente al proceso enseñanza-aprendizaje, metodologías, planificación y evaluación de la enseñanza, capacitación de personal y otros aspectos que afectan el desarrollo de la educación como tal. 
Dentro de esta dimensión pedagógica, algunas de las funciones que deben desempeñar el gestor o gestora, planteadas por García, Rojas y Campos (2002), son:

- Explicitar las concepciones acerca del aprendizaje, el conocimiento y el rol docente como marco teórico referencial que dé sustento al proyecto pedagógico.

- Explicitar los criterios para hacer las programaciones didácticas: relación de contenidos, diseños de objetivos y estrategias, organizar el tiempo y el espacio.

- (...) Gestar la capacitación.

- (...) Evaluar permanentemente la calidad del proyecto pedagógico en procesos y resultados.

- Impulsar proyectos creativos e innovadores. (pp. 21-22)

Es por lo tanto, menester del gestor o gestora la planificación, ejecución y evaluación de todos los procesos relativos al área pedagógica, comprendiendo la conceptualización de los ejes que la definen, las determinaciones estratégicas que se desarrollen, las capacitaciones que se requieran y la construcción de espacios para la creatividad y la innovación que este ámbito implique. Se requiere de un liderazgo que asuma, de manera activa y permanente, el área pedagógica, desde la claridad de su trascendencia dentro del funcionamiento integral de la organización escolar.

\section{Innovación educativa}

Por innovación se entiende la acción de incorporar algo nuevo en una realidad existente, lo cual transforma su ser y su funcionamiento, de manera que los efectos resultantes sean mejores (Rivas, 2000). La innovación implica, por lo tanto, una acción de transformación que apunta a la mejora de los resultados por los cuales se trabaja.

En el área específica de la educación, la innovación se puede definir como:

La posibilidad de cambiar los ejes fundamentales de la escuela, es romper con lo viejo, para buscar un nuevo equilibrio, destinado a alcanzar mayores logros, mayores niveles de calidad. Se trata de un proceso permanente, espiralado, complejo y contradictorio, nunca libre de conflictos. Innovar es así, romper con los aspectos estructurales de la escuela, es producir ideas nuevas y recrear las viejas, es un acto de creatividad permanente. (Aguerrondo et ál., 2006, p. 46)

Desde esta perspectiva, innovar comprende el todo de la estructura organizativa, como proceso integral, que surge desde los fundamentos mismos que definen a la educación. Su objetivo se centra en la cantidad y la calidad de los logros que se persiguen, y se genera a partir de lo existente, es, por lo tanto, un proceso de destrucción creadora. 


\section{Tipología de las innovaciones}

Se definen diversas clasificaciones en lo referente a las innovaciones. Rivas (2000) determina como criterios clasificatorios, elementos tales como los componentes, el modo de realización, la amplitud de la innovación y la intensidad del cambio propuesto.

Las innovaciones según los componentes, se definirán de acuerdo con la cantidad de elementos que afecte. Así, se pueden señalar innovaciones propias a instalaciones escolares, definiciones de roles o las relaciones entre las partes. Las innovaciones según el modo de realización, incluyen las categorías sustitución, alteración, reestructuración y reorientación en los valores, entre otras, referentes todas a la forma en que se ejecutan los procesos innovadores. Las categorías que comprende la clasificación por amplitud son definidas por la singularidad o pluralidad de quienes llevan a cabo la innovación, de manera que variarán según sean muchas o pocas las personas incluidas.

La clasificación de las innovaciones según la intensidad comprende la dimensión del cambio que se genere en el rol del docente y su consiguiente desempeño pedagógico y relacional. Dentro de estas se definen las adicionales y las fundamentales.

Las innovaciones adicionales transforman procedimientos sin modificar el rol básico docente, implican mejoras o cambio en el método pedagógico utilizado, pero sin transformarlo esencialmente. Las innovaciones fundamentales, por su parte, comprenden transformaciones en el rol docente, transformando el comportamiento educativo en las diferentes áreas y dimensiones que incluye el sistema educativo.

Este tipo de innovación incluye, según señala Rivas (2000):

Modificación en los objetivos e intenciones, en los procesos educativos y las estructuras escolares. Implican alteraciones en la metodología, en los contenidos educativos, en las relaciones interpersonales, en la toma de decisiones, en el clima escolar, incluyendo el cambio en los valores y la cultura de la institución escolar. (p. 60)

Partiendo de estas innovaciones se genera un proceso enseñanza- aprendizaje diferente, que contribuye a producir seres humanos desde procesos de pensamiento y valores diferentes, posibilitando la incorporación sociocultural y económica, de forma crítica, constructiva, creativa y solidaria, alejándose de los principios de competitividad, egocentrismo y sobrevaloración del consumo que produce la lógica globalizada de mercado que define actualmente el proceso educativo.

\section{Métodos pedagógicos}

Las innovaciones fundamentales se generan en el campo de la determinación pedagógica, ya que es desde allí donde todo el quehacer educativo es transformado. 
Como las dos principales corrientes que caracterizan los sistemas educativos actuales, se pueden identificar, según define Boggino (2007), las pedagogías transmisivas, de corte conductista y neoconductista, que determina al sujeto cognoscente como"un simple organismo reactivo $y$, por lo tanto, desconsidera toda otra actividad cognoscitiva, funda la enseñanza en la mera presentación reiterada de contenidos" (p. 19), y, las corrientes pedagógicas participativas, dentro de las cuales se ubican las constructivistas, que parten del principio de que el sujeto cognoscente construye su propio conocimiento, implicando, para el mismo, un rol activo y determinante dentro del proceso.

De manera tal que el paradigma que plantean las pedagogías participativas no se limita a estrategias didácticas, del corte de recursos técnico-metodológicos aislados, sino que implica la totalidad de las acciones que realiza el o la docente, desde una clara intencionalidad pedagógica (Boggino, 2007).

Se parte, para el constructivismo, desde una acción didáctica donde el conflicto y el diálogo son parte inminente del quehacer educativo y del relacionamiento entre las partes, que transforma el rol tradicional otorgado a docentes y a estudiantes.

Se posibilita, desde este paradigma, la construcción de:

Una escuela crítica y alternativa, al tiempo que responden a muchas de las cuestiones relativas a cómo enseñar para que los alumnos aprendan y, sobre todo, para que "aprendan a aprender". La perspectiva del constructivismo nos permite, pues, sostener la propuesta de una didáctica radical de pensamiento, centrada en el logro de objetivos socialmente relevantes, profundamente respetuosa con la personalidad del alumno y también concordante con las exigencias del conocimiento científico. (Barberá, Bolívar, Calvo, Coll, Fuster, García et ál. 2000, p. 97)

Por esta caracterización es que las innovaciones dentro del área pedagógica, se clasifican dentro de las fundamentales, respondiendo a las condiciones del contexto y a sus demandas, y a la responsabilidad de favorecer el desarrollo de cada ser humano desde su condición particular. Es, en síntesis, implementar modelos pedagógicos que hagan posible una educación significativa, desde lo particular y desde lo social.

\section{Papel docente}

Las innovaciones son procesos ejecutados, primordialmente, por docentes. Al respecto, Tedesco (2009), en referencia a los resultados obtenidos por las reformas educativas regionales de América Latina, apunta que:

Sin dejar de reconocer la importancia tanto de las variables del contexto socio-económico como las de tipo institucional, adquiere mayor relevancia la hipótesis según la cual para que 
las reformas "lleguen al aula"y se modifiquen las relaciones y los resultados de aprendizaje, la variable clave es el docente, sus métodos, sus actitudes y sus representaciones. Desde este punto de vista, la nueva agenda coloca en un lugar central las preguntas acerca de quién y cómo se enseña. (p. 809)

El personal docente es fundamental para el alcance de innovaciones. Si bien es cierto no son los únicos actores involucrados en los procesos innovadores, su rol se concretiza en acciones y procesos dentro del aula, expresando la operacionalización del proceso innovador.

La génesis de la actitud innovadora en docentes, puede corresponder a motivaciones del tipo interno o externo (Rivas, 2000). Se ubican en la categoría de los impulsores internos aquellos propios a la personalidad de la o el docente, desde un comportamiento proactivo que genera la actitud innovadora. Relativos a los impulsores externos, se incluyen los que resultan de exigencias o influencias del entorno y los del tipo reactivo, producidos como respuesta a problemas o situaciones de conflicto.

Entre los aspectos en los cuales las y los docentes deben adoptar una posición y tomar decisiones para generar innovación pedagógica, se pueden señalar, según apunta Boggino (2007), los siguientes:

- La estructura de participación y formas de comunicación dentro del aula

- La forma de presentación de los contenidos, atención a la significatividad lógica del material y la condición integral de estudiantes

- Las relaciones entre objetivos e intencionalidad educativa, desde los procesos y resultados

- Los criterios e instrumentos de evaluación a implementar

- La organización espacial en el aula

- El estilo propio y personal de enseñanza

- La forma de ayuda pedagógica a desarrollar

La posición adoptada en cada uno de estos aspectos evidencia el planteamiento pedagógico desde el cual, el o la docente desarrolla el proceso enseñanza-aprendizaje, y expresa las innovaciones experimentadas desde este ámbito.

\section{Metodología}

Este trabajo se enmarca en la categoría de investigación cuantitativa, por cuanto establece el conocimiento de la realidad de un fenómeno, mediante la recolección de datos, que son 
analizados desde la relación con los objetivos propuestos. Se plantea como descriptivo, ya que responde a la intencionalidad de definir las propiedades determinantes del fenómeno analizado, específicamente las innovaciones pedagógicas, de manera tal que se logre identificar cuáles son las acciones que desde la gestión se realizan para implementar innovaciones pedagógicas, las estrategias de seguimiento en estas implementaciones y la forma práctica en que, desde las aulas, los y las docentes ejecutan esas innovaciones en las organizaciones educativas seleccionadas.

La unidad de estudio la conforman 14 docentes que laboran en la Escuela Nueva Laboratorio Emma Gamboa de la Universidad de Costa Rica, escuela perteneciente al sistema educativo público, y 20 docentes pertenecientes al Instituto Educativo Moderno, escuela del sistema educativo privado, además de las dos directoras que laboran en cada una de las escuelas. Ambas organizaciones se caracterizan por contar con filosofías institucionales que priorizan la innovación educativa.

Los instrumentos utilizados para la obtención de la información requerida son un cuestionario dirigido a docentes y otro dirigido a las directoras de ambos centros. Estos cuestionarios se estructuraron de acuerdo con las categorías de análisis definidas, a saber:

- Mecanismos aplicados por la gestión para la implementación de innovaciones pedagógicas

- Estrategias de innovación pedagógica desarrolladas por docentes

- Acciones de seguimiento, aplicadas desde la gestión, en el desarrollo de innovaciones pedagógicas 
Tabla 1

\section{Categorías de análisis e indicadores a desarrollar en la investigación}

\begin{tabular}{|c|c|}
\hline Categoría de análisis & $\begin{array}{l}\text { Mecanismos aplicados por la gestión para la implementación de innovaciones } \\
\text { pedagógicas }\end{array}$ \\
\hline Indicadores & $\begin{array}{l}\text { - } \quad \text { Análisis conjunto, docentes y dirección, de conceptualizaciones de enseñanza y } \\
\text { aprendizaje. } \\
\text { - } \quad \text { Revisión de rol de docente y de estudiante en el proceso educativo. } \\
\text { - } \quad \text { Análisis y discusión de criterios imperantes en programaciones didácticas, del tipo } \\
\text { revisión de contenidos y estrategias. } \\
\text { - } \quad \text { Espacios para la capacitación en el área de metodologías pedagógicas. } \\
\text { - } \quad \text { Procesos evaluativos del área pedagógica, en los procesos y los resultados. } \\
\text { - } \quad \text { Estímulo de generación de proyectos pedagógicos innovadores. } \\
\text { - Posibilidad de modificación de objetivos que determinan el proceso educativo. } \\
\text { - } \quad \text { Oportunidad de cambio en contenidos educativos. } \\
\text { - Posibilidad de transformación en las metodologías pedagógicas utilizadas. }\end{array}$ \\
\hline Categoría de análisis & Estrategias de innovación pedagógica desarrolladas por docentes \\
\hline Indicadores & $\begin{array}{ll}\text { - } & \text { Definición del proceso educativo como gestor de seres humanos integrales. } \\
\text { - } & \text { Posibilidades de participación de estudiantes durante rutina escolar y en la } \\
\text { determinación de aspectos propios del proceso educativo. } \\
\text { - } \quad \text { Formas de comunicación entre docentes y estudiantes y entre estudiantes. } \\
\text { - } \quad \text { Desarrollo de aprendizaje significativo en cada área por ejecutar. } \\
\text { - } \quad \text { Congruencia entre acciones desarrolladas en el aula y los objetivos que rigen el } \\
\text { - } \quad \text { proceso. } \\
\text { - } \quad \text { Organización espacial del aula y otros espacios de la escuela que posibiliten la } \\
\text { - } \quad \text { Análisis de estilo de enseñanza propio frente a lineamientos de pedagogías } \\
\text { - } \quad \text { Usorticipativas. }\end{array}$ \\
\hline Categoría de análisis & $\begin{array}{l}\text { Acciones de seguimiento, aplicadas desde la gestión, en el desarrollo de } \\
\text { innovaciones pedagógicas }\end{array}$ \\
\hline Indicadores & $\begin{array}{l}\text { - Frecuencia de la evaluación o medición del avance de las innovaciones } \\
\text { pedagógicas implementadas. } \\
\text { - } \quad \text { Medición de cambio en el funcionamiento pedagógico de docentes. } \\
\text { - } \quad \text { Valoración de novedades implementadas en la }\end{array}$ \\
\hline \multirow[t]{2}{*}{ Categoría de análisis } & $\begin{array}{l}\text { Acciones de seguimiento, aplicadas desde la gestión, en el desarrollo de } \\
\text { innovaciones pedagógicas }\end{array}$ \\
\hline & $\begin{array}{l}\text { - } \quad \text { pedagogía. } \\
\text { - } \text { Comprobación de mejoras aportadas por implementaciones, desde los procesos } \\
\text { - } \quad \text { Cotejo de mejoras producidas por innovaciones en ejecución, desde las estructuras. } \\
\text { - Generación de cambios producto de evaluaciones realizadas. } \\
\text { Participación de diferentes actores en los procesos de valoración de las } \\
\text { innovaciones. }\end{array}$ \\
\hline
\end{tabular}

Nota: Elaboración propia, 2014. 


\section{Resultados, análisis y discusión}

\section{Mecanismos aplicados desde la gestión para la implementación de innovaciones pedagógicas}

En esta área García et ál. (2002), señalan la necesidad de"explicitar las concepciones acerca del aprendizaje, el conocimiento y el rol docente como marco teórico referencial que dé sustento al proyecto pedagógico" (p. 22). Este proyecto pedagógico puede ser definido unilateralmente por alguno de los actores del sistema, o idealmente, desde un proceso de gestión participativa, puede ser elaborado de forma conjunta con docentes, quienes son los y las ejecutoras finales de todo el planteamiento educativo.

Partiendo de esa conceptualización, pasando por los roles que asume cada actor del proceso y llegando hasta el favorecimiento de las revisiones del accionar establecido, todo determina la priorización que desde la gestión se dé a las innovaciones pedagógicas y la posibilidad real de que estas se ejecuten.

En esta categoría de análisis los criterios externados por docentes de la Escuela Nueva Laboratorio Emma Gamboa, señalan mayoritariamente que los mecanismos de implementación de innovaciones aplicados por la gestión se dan a veces, presentando aquí un $58 \%$ de las respuestas. Así mismo, apuntan que los espacios para capacitación en el área de metodologías pedagógicas así como el análisis conjunto entre docentes y dirección de las conceptualizaciones del proceso enseñanza-aprendizaje, y el análisis y discusión de criterios imperantes en programaciones didácticas (revisión de contenidos y estrategias), son elementos siempre presentes desde la gestión de la escuela.

Esta misma población de la investigación apunta con frecuencia a veces, los elementos: posibilidad de modificación de objetivos, oportunidad de cambio en contenidos, procesos evaluativos delárea pedagógica, y posibilidad de transformación en las metodologías pedagógicas.

A la pregunta abierta acerca de las estrategias desarrolladas desde la gestión, para favorecer la implementación de innovaciones pedagógicas, los criterios externados por docentes apuntan, en repetidas ocasiones, la existencia de espacios de capacitación, expresando: capacitación constante y capacitación continua del mejoramiento de la profesión, en busca de la actualización docente.

Otro de los aspectos señalados es el relativo a sistematización de los procesos desarrollados en las aulas: sistematización de nuevas técnicas pedagógicas aplicadas para una posterior valoración y se socializan ventajas y desventajas de procesos de innovación realizados con estudiantes.

Otras de las respuestas brindadas refieren a la posibilidad de aplicación de estrategias 
novedosas, aprendizaje cooperativo y el trabajo en equipo, señalando: libertad para innovar y crear, fortalecer por medio de actividades, el aprendizaje cooperativo con proyectos grupales y favorecer el trabajo en equipos como forma de integración en el aula (docentes, guías y técnicos).

En cuanto a la dirección del centro educativo, en esta misma categoría de investigación, señala que mayoritariamente su gestión presenta siempre los indicadores consultados, aunque coincide con los resultados mostrados por docentes con mayor porcentaje en la frecuencia $a$ veces.

Los criterios externados por la dirección a la pregunta abierta acerca de las estrategias que desarrolla para favorecer la implementación de innovaciones pedagógicas, refieren a: toma de decisiones con aporte de los diferentes actores, observación participativa de lecciones y análisis de las prácticas tradicionales en la escuela.

Por su parte, las personas docentes del Instituto Educativo Moderno, en esta categoría de análisis presentan mayoritariamente las respuestas en la frecuencia a veces, con un $55 \%$ de estas. Así mismo señalan dentro de la categoría siempre, los indicadores: análisis conjunto, docentes y dirección, de conceptualizaciones de enseñanza-aprendizaje así como análisis y discusión de criterios imperantes en programaciones didácticas (revisión de contenidos y estrategias).

En la frecuencia a veces, los indicadores que expresan mayores porcentajes son: posibilidad de modificación de objetivos que determinan el proceso educativo, oportunidad de cambio en contenidos educativos, espacios para la capacitación en el área de metodologías pedagógicas y posibilidad de transformación en las metodologías pedagógicas utilizadas.

En este mismo análisis, el personal docente señala algunos indicadores en la frecuencia nunca, a saber: revisión del rol de docente y estudiante en el proceso educativo, espacios para la capacitación en el área de metodologías pedagógicas y procesos evaluativos del área pedagógica, en los procesos y los resultados.

A la pregunta abierta acerca de las estrategias desarrolladas desde la gestión, para favorecer la implementación de innovaciones pedagógicas, los criterios señalan que una de las estrategias utilizadas es la toma de decisiones de forma conjunta entre la dirección y el personal docente, externando: Se respetan, toman en cuenta y discuten constantemente, las ideas y opiniones de todo el personal de trabajo y se mantiene una comunicación constante y asertiva entre el personal y la gestión.

Otro de los aspectos señalados es el referente a libertad en diversos aspectos del proceso educativo: hay libertad de planeamiento, libertad en la toma de decisiones al cuerpo docente y libertad en proceso evaluativo.

Asímismo, se resalta la adquisición y uso de tecnología. Algunas de las respuestas señaladas son: mucho interés por el uso de la tecnología, computadora, pizarra interactiva, proyector y se ha 
hecho inversión al respecto y favorecer el uso de nuevas tecnologías para propiciar un cambio.

Por su parte, la dirección señala mayoritariamente que siempre se dan mecanismos de implementación de innovaciones pedagógicas, concordando con docentes en indicadores que muestran una inclinación organizacional hacia el trabajo en equipo y la construcción conjunta de elementos fundamentales en el proceso educativo.

Los criterios brindados por la dirección a la pregunta abierta acerca de las estrategias que desarrolla para favorecer la implementación de innovaciones pedagógicas, refieren a: presentación y discusión con equipo de coordinaciones, presentación y discusión con docentes e integración de las ideas de los docentes a la propuesta.

Los aspectos mostrados en las respuestas de docentes y direcciones de ambos centros educativos permiten resaltar algunos elementos, desde la claridad de la necesidad de contribuir a la formación de seres humanos que no solamente se integren a la sociedad a la que pertenecen, sino que se involucren en la transformación de la misma, el proyecto educativo debe brindar herramientas que estimulen procesos de pensamiento, análisis y funcionamiento que posibiliten esas características. Es por ello que la pedagogía, como medio que permea todas las áreas y relaciones dentro del sistema, debe ser analizada y desarrollada cuidadosamente, nunca dejada al azar o definida sin la fundamentación filosófica organizacional.

Innovar, comprendido como proceso integral que afecta todo el accionar de la organización, debe ser un objetivo claro y teleológico para la gestión, planificado y establecido de la forma más efectiva, de manera que asegure el logro de objetivos superiores, que trasciendan el alcance de metas de rendimiento académico, para apuntar al desarrollo integral de los y las estudiantes. La claridad y determinación de la gestión en esta acuciosa labor, marca la diferencia entre el nivel de avance que se pueda lograr.

Así, el análisis y discusión de las conceptualizaciones desde donde se origina y direcciona el proceso enseñanza-aprendizaje, al igual que los contenidos y estrategias que rigen las programaciones didácticas son características que aseguran la unidad de prioridades y objetivos por alcanzar entre la gestión y el personal docente. Estos indicadores son fundamentales en la implementación de innovaciones pedagógicas, ya que permiten mantener la necesaria revisión constante del quehacer educativo.

Trascender las innovaciones adicionales, identificadas como la novedad o cambio en un área particular, para permitir generar las innovaciones fundamentales, del tipo pedagógico, requiere la posibilidad de efectuar"modificación en los objetivos e intenciones, en los procesos educativos y las estructuras escolares" (Rivas, 2000, p. 60). De allí, que el espacio para análisis de elementos fundamentales, tales como la conceptualización y base teórica desde donde parte el modelo educativo propio junto con todos los elementos que lo determinan, es indispensable. 
La flexibilidad que caracteriza la fase inicial del curso lectivo debe mantenerse a lo largo de su desarrollo. Las innovaciones pedagógicas requieren de capacidad de cambio como una condición permanente, desde el inicio y hasta la etapa final de un período educativo.

La posibilidad de modificar diferentes elementos del proceso está directamente relacionada con la implementación de innovaciones. Al respecto, Pérez (1998) señala como una de las funciones de la gestión "el proceso de estimular y dinamizar el desarrollo del currículo en sus distintas fases y etapas (investigación, programación, instrumentalización, ejecución y evaluación)" (p.10), desde donde se posibilitarían planteamientos de transformación a lo largo del curso lectivo en las áreas que lo ameriten, sin restringirse a las revisiones y proyecciones de inicio del mismo. Queda establecido que el espacio para generar esta dinamización permanente, con los procesos particulares que amerite, corresponde al gestor o gestora de la educación, complementando las revisiones y construcciones iniciales, las cuales pueden resultar insuficientes para mantener el carácter flexible e innovador.

Otro aspecto que debe analizarse es la necesidad de espacios para la capacitación, insumo indispensable dentro de una dinámica innovadora, concebida como un acto de creatividad permanente (Aguerrondo et ál., 2006). Es de suponer que esta creación constante requiere ser enriquecida con elementos externos, producto de investigaciones y propuestas novedosas, que deben ser socializadas con el equipo docente. Conocer y analizar lo que sucede en otros ambientes y realidades puede generar las ideas precisas para innovar, proceso liderado desde la gestión, como ente investigador que aporta recursos para atender las necesidades de la organización a su cargo.

En lo concerniente a los roles otorgados a los actores del proceso educativo, Freire, citado por Rodríguez et ál. (2007), resalta que la educación es "primeramente comunicación, diálogo entre iguales que se relacionan; no es meramente una acción comunicativa que tiende al consenso sino que es una acción estratégica que apunta a la consecuencia de un fin, que es la transformación del mundo" (p. 136). Para alcanzar un proceso comunicativo respetuoso y asertivo como el señalado, es menester revisar los roles designados ya que estos son producto de la conceptualización ontológica en la cual se basa el proyecto, del cual se deriva, asimismo, la propuesta pedagógica sostenida.

En lo referente a la evaluación de procesos y resultados, ésta es indispensable ya que muestra el avance de los objetivos durante el proceso y los alcances finales del mismo, razón por la cual debe estar presente desde una metodología que se plantee la innovación.

Al respecto, Pérez (1998) apunta que entre las responsabilidades de la gestión se encuentra "estimular y dinamizar el desarrollo del currículo en sus distintas fases y etapas (investigación, programación, instrumentalización, ejecución y evaluación)" (p. 10), por lo que el proceso evaluativo es determinante en el desarrollo del proyecto educativo. Evaluaciones conjuntas, 
periódicas y claramente definidas, deberán ser determinadas desde la gestión, en procura del logro de un proceso innovador desde lo pedagógico y, por ende, de una educación de calidad.

\section{La ejecución de innovaciones pedagógicas desarrolladas por docentes}

La implementación del proyecto educativo particular recae de forma directa en el cuerpo docente, el cual tiene la función de trasladar a hechos todo el planteamiento filosófico organizacional. En lo relativo a las innovaciones pedagógicas, los y las docentes son actores determinantes en el alcance de estas.

El ejercicio de un rol activo y pensante por parte de los y las maestras, es posibilitado desde la gestión al "hacer partícipes activos a los docentes en la toma de decisiones curriculares y de las innovaciones en el arte de mediar y el de aprender" (Consejo Superior de Educación, 1994, p. 10), asegurando el involucramiento real en un proyecto construido en conjunto, donde los aportes, ideas y experiencias de todos los actores, enriquece el producto final.

Este planteamiento deja de lado la conceptualización del cuerpo docente como un ente meramente ejecutor de un currículo rígido, previamente determinado y a través de una metodología predeterminada, que no cuenta con posibilidad de aportar al avance organizacional y donde las innovaciones no son producto de la investigación ni del análisis compartido. Se apunta, por lo tanto, a una gestión que facilite la participación y potencie el talento humano con que se cuenta, de manera que el liderazgo ejercido promueva estas elaboraciones.

La información obtenida de los y las docentes de la Escuela Nueva Laboratorio Emma Gamboa acerca de estos tópicos muestra frecuencia mayoritaria en las respuestas siempre, registrando $59 \%$ de estas. Así, los indicadores presentados con mayor porcentaje son los concernientes a participación y posibilidad de decisión de estudiantes en la rutina diaria, comunicación asertiva y continua entre docentes y estudiantes, desarrollo de aprendizaje significativo, congruencia entre acciones desarrolladas en el aula y los objetivos que rigen el proceso y, organización espacial del aula y otros espacios de la escuela que posibiliten la construcción del aprendizaje.

En este análisis, los y las docentes apuntan los indicadores: criterios de evaluación correspondientes a pedagogía utilizada y uso de ayudas pedagógicas correspondientes a metodologías participativas, con respuestas en la frecuencia nunca, lo cual evidencia necesidad de revisión en ambas áreas.

Los criterios expresados por docentes a la pregunta abierta acerca de las estrategias implementadas en el aula, los y las docentes señalan con mayor frecuencia las siguientes: el niño propone tema a estudiar y debe respetarse la decisión de la mayoría, construyen las normas de convivencia y, el estudiante brinda ideas. 
Además de estas acciones, apuntan el trabajo en equipo o aprendizaje colaborativo, expresando: procesos grupales que promuevan el aprendizaje cooperativo, participación activa en procesos grupales y, colaboración entre pares de diferentes edades.

Otra de las acciones aplicadas es la relativa a la ejecución de aprendizaje significativo, señalando: propiciar un aprendizaje significativo y se toma en cuenta los intereses y necesidades como referencia para el trabajo de aula.

En esta misma categoría de análisis la dirección, coincidentemente, responde mayoritariamente siempre a la ejecución de innovaciones pedagógicas por parte de docentes, presentando $67 \%$ de las respuestas. Se observa que las estrategias desarrolladas por docentes en las prácticas educativas para ejecutar innovaciones pedagógicas, muestra un balance positivo, presentando una amplia cantidad de presencia, según lo expresan docentes y dirección.

En la consulta abierta, la dirección indica como estrategias que utilizan las personas docentes: desarrollo de unidades integradas, giras pedagógicas, proyectos ambientales, desarrollo de procesos comunicativos (actos cívicos organizados por los chicos, exposiciones de aprendizajes logrados).

Por su parte, la información brindada por los y las docentes del Instituto Educativo Moderno, en lo referente a estrategias empleadas por docentes para ejecutar innovaciones pedagógicas muestran frecuencia alta en las respuestas siempre, presentando un $68 \%$ de estas.

La mayoría de los indicadores estudiados señalan frecuencia mayor a un 50\%. De manera tal, que los indicadores que registran los mayores porcentajes son: organización espacial del aula y otros espacios de la escuela que posibiliten la construcción del aprendizaje, congruencia entre acciones desarrolladas en el aula y los objetivos que rigen el proceso, uso de ayudas pedagógicas correspondientes a metodologías participativas y, definición del proceso educativo como gestor de seres humanos integrales.

En la categoría nunca se registran dos indicadores: análisis de estilo de enseñanza propio frente a lineamientos de pedagogías participativas $y$, comunicación asertiva y continua entre docentes y estudiantes.

Con respecto a la pregunta abierta acerca de las estrategias desarrolladas con mayor frecuencia, los y las docentes del Instituto Educativo Moderno apuntan la implementación de programas complementarios tales como: programa de educación ambiental, programa de méritos y deméritos y programas de educación para la paz.

También señalan acciones que estimulan la autonomía, expresando: promoción de la autonomía y la independencia a partir del autoconocimiento y la libertad con responsabilidad y proceso de manejo de conflictos de forma asertiva. 
Otro aspecto que se presenta es el relacionado con el estímulo de aprendizaje colaborativo, señalando: el estudiante debe estar consciente que es responsable de su propio aprendizaje, al mismo tiempo que tiene que compartir y/o adquirir el conocimiento con sus pares, o trabajo en equipo.

Así mismo se señalan las acciones tendientes a la promoción de los valores, registrando: valor o pensamiento del día: escrito en la pizarra y analizado por parte de estudiantes y aplicar cotidianamente fortalecimiento de valores.

En este mismo análisis, la dirección responde mayoritariamente siempre a las ejecuciones de innovaciones por parte de docentes, presentando un $56 \%$ de las respuestas. Así mismo, en contraste con los y las docentes, la dirección apunta varios indicadores con frecuencia mayoritaria a veces, mismas que son señaladas como siempre presentes por ellos y ellas.

A la pregunta abierta en este apartado, la dirección señala los elementos: plan de méritos y deméritos, apoyo del departamento de psicología (dinámicas), evaluación de la conducta, programas extracurriculares: ambiental, educación para la paz, etc., y celebraciones y fechas institucionales (efemérides, festival deportivo, etc.).

Una vez analizada la información brindada por los sujetos de investigación de ambos centros educativos, cabe resaltar algunos aspectos importantes.

Entre los elementos señalados como sujeto de mejora, se identifican algunos propios a la forma de enfrentar la labor docente, específicamente, la revisión del estilo propio de enseñanza y las formas de evaluación aplicadas.

Las innovaciones pedagógicas son posibles desde una experimentación de la labor docente que comprenda análisis constante del desempeño alcanzado, de manera que se asegure la congruencia entre lo planteado teóricamente y lo desarrollado de forma práctica. La función de la gestión a este respecto comprende la evaluación constante de la calidad del proyecto pedagógico que se ejecuta, comprendiendo tanto los procesos como los resultados (García et al., 2002), para lo cual se vuelve indispensable la confrontación de lo realizado a la luz de experiencias externas, desde donde puedan extraerse insumos para el análisis organizacional.

Otra área identificada como susceptible de ser mejorada apunta al lugar otorgado al estudiantado, incluyendo áreas como la posibilidad de estos de decidir, el tipo de comunicación que se establece con ellos y ellas, además del desarrollo de un aprendizaje significativo.

La UNESCO (2005), al resaltar los objetivos que la educación debe buscar, plantea que:

Hay tres principios que tienden a ser ampliamente compartidos y pueden resumirse de la siguiente manera: necesidad de una mayor pertinencia, necesidad de una mayor equidad en el acceso y los resultados, y necesidad de respetar los derechos de la persona como es debido. La mayoría de los que han reflexionado a nivel internacional sobre la cuestión estiman que esos principios no solo orientan e informan los contenidos y procesos 
educativos, sino que además representan objetivos sociales más generales a los que la educación debe contribuir. (pp. 33-34)

Este respeto que debe incluirse dentro del proceso educativo, pasa por el otorgamiento de un lugar a cada actor, incluidos los y las estudiantes. Respeto que comprende posibilidad de expresarse y de que sea tomado en cuenta ese parecer, además de participar de procesos comunicativos asertivos, donde se visibilicen los intereses y características de la población por atender, por demás, única forma de lograr una educación significativa. De manera tal que el rol que se le asigne al estudiante es determinante en la consecución de una educación que busque innovación desde el plano pedagógico, visionando además el logro de objetivos sociales que trasciendan a lo meramente académico o del área de contenidos curriculares.

\section{El seguimiento en las innovaciones pedagógicas, desde la gestión}

Un proceso educativo que plantee la innovación como un objetivo por alcanzar, exige para la gestión un proceso sostenido que permita llevar hasta el final el desarrollo de las ideas iniciadas. Los buenos planteamientos deben ser acompañados y guiados desde la gestión de manera que se conviertan, al menos, en aquello en lo que fueron pensados.

Dentro de una dinámica compleja como la educativa, la generación de ideas es solamente el principio de un proceso por desarrollar, y no comprende, en sí misma, la posibilidad de asegurar éxito en la tarea iniciada. Corresponde a la gestión establecer las estrategias que posibiliten ese logro, desde el seguimiento y valoración constante a lo largo de todo el proceso.

A este respecto, la información obtenida de docentes en la Escuela Nueva Laboratorio Emma Gamboa señala mayoritariamente la frecuencia a veces en los indicadores de seguimiento de las innovaciones pedagógicas desde la gestión, con un 54\% de las respuestas. De igual forma presentan con siempre presentes los indicadores: generación de cambios producto de evaluaciones realizadas y participación de diferentes actores en los procesos de valoración de las innovaciones.

En lo referente a la frecuencia nunca, seis de los siete indicadores muestran respuesta, siendo los de mayor frecuencia: evaluación o medición del avance de las innovaciones pedagógicas implementadas, comprobación de mejoras aportadas por implementaciones, desde los procesos generados y cotejo de mejoras producidas por innovaciones en ejecución.

El personal docente expresa a la pregunta abierta acerca de las acciones que desarrolla la gestión para darle seguimiento a las innovaciones pedagógicas aplicadas, mayoritariamente, criterios referentes al análisis y evaluación, señalando: análisis de los procesos y actividades realizadas en la escuela, se comenta de manera individual cada proyecto: viabilidad, reuniones de personal: revisión y análisis de actividades. 
También se expresan ideas referentes a actualizaciones y capacitaciones, señalando: coordinardiversos tipos de capacitaciones sobreel tema o cursos de actualizaciones ycapacitaciones.

Otra de las estrategias descritas son las relativas a las revisiones de las prácticas laboratorio, una de las estrategias propias de la organización que posibilita el aprendizaje autoconstruido, significativo y colaborativo. Indicaron: análisis de los diferentes procesos o prácticas laboratorio y énfasis de las prácticas laboratorio, que o deben faltar.

Las respuestas de la dirección, por su parte, registran mayoritariamente siempre, con una frecuencia de 57\%, lo que no es coincidente con el análisis de los y las docentes. Así mismo, la frecuencia nunca, no presenta ninguna respuesta por parte de la dirección, en contraposición con lo contestado por docentes.

En este mismo apartado, a la pregunta abierta, la dirección responde que aplica las siguientes estrategias de seguimiento: visitas periódicas a los grupos y sesiones de análisis prácticas de laboratorio que no deben faltar.

Los datos brindados por docentes y dirección muestran, en forma general, un nivel bajo en la frecuencia en lo referente al seguimiento generado hacia las innovaciones, en contraste con las otras dos área analizadas.

En cuanto a la información brindada por docentes del Instituto Educativo Moderno, las respuestas señalan, con un 43\%, mayoritariamente a veces presentes las acciones de seguimiento en las innovaciones pedagógicas, desde la gestión. De igual forma, apuntan como siempre presentes los indicadores: generación de cambios producto de evaluaciones realizadas y cotejo de mejoras producidas por innovaciones en ejecución, desde las estructuras.

En lo referente a la opción nunca, los siete indicadores presentan alguna frecuencia de respuesta, condición que no se presenta en las áreas anteriormente analizadas. Los que mayor porcentaje de respuesta registran son: frecuencia de la evaluación o medición del avance de las innovaciones pedagógicas implementadas, participación de diferentes actores en los procesos de valoración de las innovaciones $\mathrm{y}$, cotejo de mejoras producidas por innovaciones en ejecución, desde las estructuras.

A la pregunta abierta acerca de los mecanismos de seguimiento a las innovaciones pedagógicas, aplicados desde la gestión, de forma mayoritaria los y las docentes señalan acciones de análisis y evaluación de los procesos, apuntando: la evaluación institucional, visitas de la dirección a las clases y análisis institucional a final de año.

También registran respuestas frecuentes en cuanto a reuniones periódicas de personal, señalando: reuniones semanales y se comparten ideas y propuestas en reuniones con el personal, basados en el desempeño o los resultados de los estudiantes tras implementar una innovación pedagógica. 
Otra de las estrategias apuntadas es la relativa a los espacios de retroalimentación, señalando: retroalimentación con autoevaluación y evaluación institucional.

Por su parte, la dirección presenta una mayor frecuencia en la opción siempre, mostrando un $71 \%$ de las respuestas. Se observa que esta área de seguimiento de las innovaciones implementadas muestra la mayor de las divergencias entre la información brindada por el personal docente y la dirección. Es esta área también, la única, de las tres investigadas, que presenta respuestas en todos los indicadores en la frecuencia nunca, por parte del personal docente.

En cuanto a la pregunta abierta acerca de estas acciones de seguimiento aplicadas a las innovaciones implementadas, la dirección apunta la aplicación de las siguientes estrategias de seguimiento: Evaluaciones con el personal docente, evaluaciones en talleres de martes y evaluaciones en análisis institucionales.

En ambas organizaciones, la divergencia mayor entre las respuestas brindadas por docentes y dirección, se da en esta categoría de análisis. Se pueden identificar aspectos comunes por mejorar en ambas escuelas.

Así, la frecuencia de las evaluaciones es un elemento determinante que puede marcar la diferencia entre el alcance total de los objetivos de un proyecto y el de solamente una etapa del mismo. A este respecto, Medina (1994), citado por Gairín (1997), plantea que la evaluación dentro del proceso educativo debe ser una reflexión valorativa y de análisis permanente, de manera que se puedan seleccionar las decisiones más adecuadas que garanticen la adaptación del currículo y todos sus componentes. Queda establecido que la evaluación debe ser desarrollada de forma constante, razón por la cual la gestión debe procurarse los medios y recursos que le permitan alcanzar esa condición de revisión permanente, desde la cual se posibilite el logro de las metas trazadas y, por ende, una educación de calidad.

Otro elemento que se registra en el análisis es el relacionado con la cantidad de actores involucrados en los procesos de medición y análisis. Partiendo de la conceptualización de que innovar implica, entre otros, cambios en las relaciones interpersonales, de manera que se produzca un cambio en los valores y la cultura organizacional (Rivas, 2000), es indispensable transformar la estructura participativa que contiene al proyecto educativo. La metamorfosis de la cultura organizacional requiere, indiscutiblemente, del involucramiento de la mayor cantidad posible de actores, de manera que los objetivos, como fin último, pero además los procesos, como medio para alcanzar aquellos, permeen toda la organización, asegurando conocimiento e involucramiento de todos y todas para alcanzar lo propuesto.

Un elemento más que debe alentar la búsqueda de la participación de la mayor cantidad posible de actores, definida de forma particular según las condiciones de cada organización, es que la visión es enriquecida desde las expectativas, conocimientos y experiencias de cada sector 
involucrado. La visión que desde la gestión se produzca, sin el aporte de otros y otras, puede resultar obsoleta, incomprendida o inadecuada. Encontrar el balance entre la visión propia y la de los demás, es tarea de la gestión, en procura de una construcción exitosa en cualquier área de crecimiento, incluida la implementación de innovaciones pedagógicas.

Con la claridad de que innovar no resulta de un proceso casual o de manera natural, la gestión amerita establecer estrategias eficientes y eficaces que aseguren a todos los actores del proceso, el análisis y atención de las circunstancias que surjan en el avance. Más que como un ojo fiscalizador, la gestión que pretenda éxito en las innovaciones pedagógicas, debe acompañar, identificar peligros, reconocer logros, tomar decisiones oportunas y redireccionar cualquier elemento que se desvíe de la trayectoria requerida para alcanzar la meta fijada.

La urgencia de respaldar acciones de seguimiento desde la gestión suele ser contrarrestada por las exigencias de funciones y requerimientos propios de la labor de dirección, cuyos objetivos y priorizaciones no necesariamente son concordantes con modelos educativos que planteen las innovaciones pedagógicas.

De forma contraria al sistema existente, que responde a la lógica económica globalizada, donde "la adopción de dichas tácticas competitivas conlleva a que las decisiones administrativas se preocupen primordialmente por fines pragmáticos y resultados inmediatos, independientemente de su valor ético y su relevancia cultural" (Sander, 2002, p. 26), las organizaciones educativas que pretendan contribuir en la formación de seres humanos críticos, creativos y autónomos, que busquen cambio en las relaciones de poder, invirtiendo en procesos y no necesariamente en valoraciones numéricas, se encuentran frente a la encrucijada de balancear sus determinaciones frente a las exigencias delegadas.

De allí que las personas gestoras apunten a proyectos innovadores, se enfrentan a las exigencias de un sistema con propósitos diferentes, cuyas exigencias laborales plantean otros usos de recursos, como el tiempo, y otros criterios de evaluación de desempeño de docentes. Por lo tanto, debe la gestión encontrar el espacio para cumplir con estos requerimientos ineludibles $y$, sustentar las innovaciones pedagógicas desde metodologías de seguimiento eficientes, de forma complementaria, sin ser tareas que se espera que ejerza.

\section{Aportes de la investigación}

La investigación desarrollada en estas organizaciones educativas muestra la validez y necesidad de evaluar el modelo educativo que se sustenta.

De forma puntual, se permite dimensionar el logro de innovaciones pedagógicas desde los siguientes ámbitos: la conceptualización de innovación de la que se parte, el desarrollo práctico de la teoría planteada (filosofía organizacional) mismo que es mediado por la unidad 
de criterios y acciones entre la gestión y docentes, y finalmente, la necesidad de mantener una evaluación sostenida del modelo pedagógico.

Identificar las áreas que afectan o potencian el avance organizacional hacia la innovación pedagógica, es posible a partir del análisis conjunto entre gestión y demás actores dentro del proceso educativo, de criterios como los definidos en el presente trabajo, los cuales permiten ubicar elementos esenciales que aseguren la innovación pedagógica.

\section{Conclusiones}

Las áreas analizadas en este trabajo responden a las estrategias de implementación de innovaciones pedagógicas por parte de la gestión, las acciones aplicadas por docentes para desarrollar estas innovaciones y las estrategias de seguimiento ejercidas por la gestión en cuanto al desarrollo de estas.

Los hallazgos encontrados en las dos organizaciones investigadas se detallan a continuación.

\section{Escuela Nueva Laboratorio Emma Gamboa de la Universidad de Costa Rica}

Según las categorías investigadas, las conclusiones correspondientes a la Escuela Nueva Laboratorio “Emma Gamboa" de la Universidad de Costa Rica, se detallan seguidamente.

- En cuanto a los mecanismos aplicados desde la gestión para la implementación de innovaciones pedagógicas: Se establece que el análisis conjunto, docentes y dirección, de conceptualizaciones de enseñanza y aprendizaje, al igual que espacios para la capacitación en el área de metodologías pedagógicas, son elementos siempre presentes desde la gestión de la organización, lo cual constituye fortalezas importantes dentro de la organización.

Por otro lado, se determina que la posibilidad de modificación de objetivos y oportunidad de cambio en contenidos, los procesos evaluativos del área pedagógica, en los procesos y los resultados, y la posibilidad de transformación en las metodologías pedagógicas utilizadas, se encuentran a veces presentes en la gestión de la escuela. Son, por lo tanto, áreas que deben ser atendidas.

Se registra diferencia en cuanto al análisis y discusión de criterios imperante en las programaciones, variando entre a veces y siempre presente. En la misma condición se presenta la revisión del rol de docentes y estudiantes, así como el estímulo de generación de proyectos pedagógicos innovadores y la posibilidad de transformación en las metodologías pedagógicas empleadas. Esta condición los ubica como elementos que requieren revisión. 
La gestión aplica otros mecanismos para implementar innovaciones, entre ellos están: la sistematización de experiencias, la posibilidad de aplicación de estrategias novedosas, el favorecimiento de aprendizaje cooperativo y el trabajo en equipo. Además, se favorece la toma de decisiones con aporte de los diferentes actores.

Algunos obstáculos que presenta la gestión para implementar innovaciones son: exceso de funciones o tareas y cambios en el puesto de dirección.

- $\quad$ En cuanto a las estrategias de innovación pedagógica desarrolladas por docentes: Entre las estrategias desarrolladas por docentes se encuentran, siempre presentes, la participación y posibilidad de decisión de estudiantes en la rutina diaria, la comunicación asertiva y continua entre docentes y estudiantes, además del desarrollo de aprendizaje significativo.

Otras estrategias desarrolladas siempre son: congruencia entre las acciones desarrolladas en el aula y los objetivos que rigen el proceso, además de organización espacial del aula y otros espacios de la escuela que posibiliten la construcción del aprendizaje.

Los criterios de evaluación correspondientes a la pedagogía utilizada, además de análisis de estilo de enseñanza propio frente a lineamientos de pedagogías participativas, son elementos presentes, a veces.

El uso de ayudas pedagógicas correspondientes a metodologías participativas es una condición poco presente dentro de la organización.

Otras estrategias desarrolladas en el aula para innovar pedagógicamente son: trabajo en equipo, aprendizaje colaborativo y desarrollo de un aprendizaje significativo.

- En cuanto a las acciones de seguimiento, aplicadas desde la gestión, en el desarrollo de innovaciones pedagógicas: La generación de cambios producto de evaluaciones realizadas es una condición siempre presente desde la gestión de la escuela.

La frecuencia de la evaluación o medición del avance de las innovaciones pedagógicas implementadas y la medición de cambio en el funcionamiento pedagógico de docentes, son características a veces existentes en la organización.

La participación de diferentes actores en los procesos de valoración de las innovaciones, al igual que la valoración de novedades implementadas en la pedagogía, la comprobación de mejoras aportadas por implementaciones, desde los procesos generados y el cotejo de mejoras producidas por innovaciones en ejecución, son valorados de forma divergente entre siempre, a veces y nunca presentes.

Otras acciones señaladas, que realiza la gestión para darle seguimiento a las innovaciones implementadas son: análisis y evaluación de actividades desarrolladas o por desarrollar, las actualizaciones y las revisiones de las prácticas laboratorio. 
Entre los obstáculos que enfrenta la gestión para dar seguimiento a las innovaciones se encuentran: insuficiencia de tiempo y recargo de funciones o tareas.

\section{Instituto Educativo Moderno}

Las conclusiones obtenidas una vez realizada la investigación en este centro educativo, se presentan a continuación.

- En cuanto a los mecanismos aplicados desde la gestión para la implementación de innovaciones pedagógicas: Se concluye del análisis conjunto, docentes y dirección, que las conceptualizaciones de enseñanza y aprendizaje, además del análisis y discusión de criterios imperantes en programaciones didácticas, del tipo revisión de contenidos y estrategias, son elementos siempre presentes en la organización.

En cuanto a la existencia de espacios para la capacitación en el área de metodologías pedagógicas, es una condición que se presenta a veces dentro de la organización.

La posibilidad de modificación de objetivos y oportunidad de cambio en contenidos es valorada de forma divergente entre siempre y a veces presente. Se concluye que es un área que debe ser trabajada.

La revisión del rol de docente y estudiante en el proceso educativo, y los procesos evaluativos del área pedagógica presentan valoraciones diferenciadas entre siempre y nunca presentes, por lo que se concluye que requieren de revisión y análisis.

Otras estrategias que aplica la gestión para implementar innovaciones pedagógicas son: libertad en diversos aspectos del proceso educativo, además de la adquisición y uso de tecnología.

Entre los obstáculos que presenta la gestión se encuentran: la falta de tiempo y dificultad en el acompañamiento y seguimiento. Asimismo se presentan limitaciones administrativas del tipo de recursos económicos.

- $\quad$ En cuanto a las estrategias de innovación pedagógica desarrolladas por docentes: Se cuenta en la organización con estrategias siempre desarrolladas por los docentes tales como: organización espacial del aula y otros espacios de la escuela que posibiliten la construcción del aprendizaje, congruencia entre acciones desarrolladas en el aula y los objetivos que rigen el proceso, y el uso de ayudas pedagógicas correspondientes a metodologías participativas.

- En cuanto a la comunicación asertiva y continua entre docentes y estudiantes, además de la definición del proceso educativo como gestor de seres humanos integrales, se muestran valoraciones diferenciadas, oscilando entre siempre y a veces presentes. Amerita esta situación que ambas condiciones sean analizadas. 
Por su parte los elementos: criterios de evaluación correspondientes a pedagogía utilizada además del análisis de estilo de enseñanza propio frente a lineamientos de pedagogías participativas, se presentan a veces.

La posibilidad de participación de estudiantes durante la rutina escolar y en la determinación de aspectos propios del proceso educativo, al igual que el desarrollo de aprendizaje significativo en cada área por ejecutar, son valorados de forma divergente, presentándose como siempre presentes y como, a veces existentes.

Otras acciones que se desarrollan en procura de innovar pedagógicamente son: programas complementarios, estimulo de la autonomía, motivación del aprendizaje colaborativo y promoción de los valores.

- En cuanto a las acciones de seguimiento, aplicadas desde la gestión, en el desarrollo de innovaciones pedagógicas: El cotejo de mejoras producidas por innovaciones en ejecución, desde las estructuras y la generación de cambios producto de evaluaciones realizadas, son elementos siempre presentes en la organización.

El resto de acciones de seguimiento aplicadas por la gestión, en lo referente a innovaciones pedagógicas implementadas, muestra gran divergencia en las valoraciones realizadas.

La valoración de novedades implementadas en la pedagogía, la medición de cambio en el funcionamiento pedagógico de docentes, la comprobación de mejoras aportadas por implementaciones, desde los procesos generados, la frecuencia de la evaluación o medición del avance de las innovaciones pedagógicas implementadas y la participación de diferentes actores en los procesos de valoración de las innovaciones, se registran como siempre, a veces o nunca presentes. Se concluye que esta área de seguimiento requiere de análisis exhaustivo para determinar sus alcances y acciones oportunas a desarrollar.

- En cuanto a otras estrategias utilizadas para ejecutar ese seguimiento, se señalan: reuniones periódicas de personal, realimentación y análisis institucionales anuales.

En lo referente a obstáculos que interfieren en el seguimiento, se encuentran: falta de tiempo y ausencia de seguimiento por parte de la gestión.

La responsabilidad de la gestión de la educación de guiar y orientar el quehacer organizacional en procura de cumplir con el objetivo de generar un proceso de calidad, implica que debe priorizarse el área pedagógica. La gestión requiere establecer procedimientos y estrategias que permitan la formación de seres humanos más preparados para enfrentar los retos cognitivos, pero igualmente los socioafectivos, de manera que se incorporen a su medio social y cultural con la capacidad de cuestionarlo y transformarlo.

Las innovaciones en el área pedagógica, fundamentales como lo son por la profundidad e integralidad que implican, deben ser parte de la agenda y función que la gestión desarrolle, 
garantizando que se alcance esa construcción significativa del proceso educativo. Se requiere de la capacidad organizacional para implementarlas, desarrollarlas en el quehacer diario de manera que permeen todas las estructuras y procesos, y darles el seguimiento apropiado para asegurar el éxito de las mismas.

En este funcionamiento, las acciones de la gestión son determinantes, razón por la cual es importante analizar el alcance logrado, identificando aciertos y situaciones por mejorar, de manera que se avance en la construcción de una cultura de innovación pedagógica.

\section{Referencias}

Aguerrondo, I., Lugo, M. T., Pogré, P., Rossi, M. y Xifra, S. (2006). La escuela del futuro Il. Cómo planifican las escuelas que innovan. Argentina: Educación-Papers Editores.

Aubert, A. y García, C. (2009). La pedagogía crítica y el éxito académico de todos y todas. TESI, 10 (3), 231-242. Recuperado de: http://www.redalyc.org/pdf/2010/201014898014.pdf

Barberá, E., Bolívar, A., Calvo, J.R., Coll, C., De Manuel, J., Fuster, J., García, M.C., Grau, A., López, A., Marrero, M., Mollá, J., Navarro, M.C., Onrubia, J., Pozo, J.I., Rodríguez F., Segura, J.M., Soler M., Teberosky, A., Torres, M.M. y Yabar, J.M. (2000). El constructivismo en la práctica. España: Editorial Laboratorio Educativo y Editorial Graó.

Boggino, N. (2007). El constructivismo entra al aula. Argentina: Homo Sapiens Ediciones.

Bunes, M., Cánovas, M. J., Ruiz, M. J. y López, R. (2009). Innovación educativa en España: un estudio descriptivo a partir de los datos en REDINED. Educatio Siglo XXI, 27, 133-168. Recuperado de: http://revistas.um.es/educatio/article/view/71121/68661

Consejo Superior de Educación. (1994). Política educativa hacia el siglo XXI. Recuperado de: http://www.oei.es/quipu/costarica/politicaeducativasigloXXI.pdf

Dengo, M. E. (2001). Desafíos, perspectivas y proyecciones del administrador educativo para el siglo XXI. Educare, 1, 51-59. Recuperado de: http://www.revistas.una.ac.cr/index.php/ EDUCARE/article/view/919

Gairín, J. (Ed). (1997). Estrategias para la gestión del Proyecto Curricular de Centro Educativo. Madrid: Institut de Ciències de l’Educació y Editorial Síntesis. 
García, N., Rojas, M. y Campos, N. (2002). La administración escolar para el cambio yel mejoramiento de las instituciones educativas. San José, Costa Rica: EUCR.

López, S. y Flores, M. (2006). Las reformas educativas neoliberales en Latinoamérica. Revista Electrónica de Investigación Educativa, 8 (1). Recuperado de: http://redie.uabc.mx/vol8no1/ contenido-flores.html

Moreno, M. (1997). Innovaciones pedagógicas. Una propuesta de evaluación crítica. Colombia: Cooperativa Editorial Magisterio.

Organización de las Naciones Unidas. (2000). Cumbre del Milenio. Declaración del Milenio. Recuperado de: http://www.un.org/spanish/milenio/ares552.pdf

Organización de las Naciones Unidas. (2012). La educación ante todo. Recuperado de: http:// www.globaleducationfirst.org/files/Education_First_SP.pdf

Organización de las Naciones Unidas para la Educación, la Ciencia y la Cultura (UNESCO). (1990). Declaración Mundial sobre Educación para todos. Marco de acción para satisfacer las necesidades básicas de aprendizaje. Recuperado de: http://www.unesco.org/education/ pdf/JOMTIE S.PDF

Organización de las Naciones Unidas para la Educación, la Ciencia y la Cultura (UNESCO). (2000). Foro Mundial sobre la Educación. Marco de acción de Dakar. Educación para todos: cumpliendo nuestros compromisos comunes. Recuperado de: http://unesdoc.unesco.org/ images/0012/001211/121147s.pdf

Organización de las Naciones Unidas para la Educación, la Ciencia y la Cultura (UNESCO). (2005). Educación para todos. El imperativo de la calidad. Recuperado de: http://unesdoc.unesco. org/images/0015/001501/150169s.pdf

Organización de las Naciones Unidas para la Educación, la Ciencia y la Cultura (UNESCO). (2011). Manual de gestión para directores de instituciones educativas. Recuperado de: http:// unesdoc.unesco.org/images/0021/002191/219162s.pdf

Pérez, R. A. (1998). La gestión curricular en las instituciones educativas. San José, Costa Rica: Ministerio de Educación Pública. 
Pinto, R. (2010). En América Latina innovar en educación es posible gracias al esfuerzo crítico de sus educadores. REXE, 17 (9), 65-83. Recuperado de: http://dialnet.unirioja.es/servlet/ articulo?codigo $=3584131$

Ramírez, L. (2012). Calidad escolar: un nuevo ethos escolar. Educación, 36 (2), 1-21. Recuperado de: http://www.redalyc.org/articulo.oa?id=44024857003

Rivas, M. (2000). Innovación educativa Teoría, procesos y estrategias. España: Editorial Síntesis, S.A.

Rodríguez, L., Marín, C., Moreno, S. y Rubano, M. (2007), Freire: una pedagogía desde América Latina. Ciencia, docencia y tecnología, 34, 129-171. Recuperado de: http://www.scielo.org. ar/pdf/cdyt/n34/n34a05.pdf

Sander, B. (2002). Políticas públicas y gestión educativa en América Latina: Discurso global y realidades locales. Pensamiento educativo, 31, 13-30. Recuperado de: http:// pensamientoeducativo.uc.cl/files/journals/2/articles/227/public/227-536-1-PB.pdf

Tedesco, J. C. (2009). Calidad de la educación y políticas educativas. Cuadernos de Pesquisa, 39 (138), 795-811. Recuperado de: http://www.scielo.br/pdf/cp/v39n138/v39n138a06.pdf

Torres, C. A. (2008). Después de la tormenta neoliberal: La política educativa latinoamericana entre la crítica y la utopía. Revista Iberoamericana de Educación, 48, 207-229. Recuperado de: http://www.rieoei.org/rie48a10.pdf 